\title{
(6) OPEN ACCESS \\ The relationship and effects of golf on physical and mental health: a scoping review protocol
}

\author{
A Murray, ${ }^{1,2}$ L Daines, $^{2}$ D Archibald, ${ }^{3}$ R Hawkes, ${ }^{4}$ L Grant, ${ }^{2}{ }^{2}$ Mutrie ${ }^{1}$
}

${ }^{1}$ Physical Activity for Health Research Centre, University of Edinburgh, Edinburgh, UK

${ }^{2}$ Usher Institute of Population Health Sciences and Informatics, The University of Edinburgh, Edinburgh, UK

${ }^{3}$ Scottish Collaboration for Public Health Research and Policy, University of Edinburgh, UK

${ }^{4}$ European Tour Golf, Virginia Water, UK

\section{Correspondence to} Dr A Murray, Physical Activity for Health Research Centre, 2.33 St Leonard's Land, University of Edinburgh, Edinburgh EH8 8, UK; docandrewmurray@googlemail. com

Accepted 20 April 2016 Published Online First 29 April 2016
CrossMark

To cite: Murray $A$, Daines L, Archibald D, et al. $\mathrm{Br} J$ Sports Med 2016;50:647-650.

\section{ABSTRACT}

Introduction Golf is a sport played in 206 countries worldwide by over 50 million people. It is possible that participation in golf, which is a form of physical activity, may be associated with effects on longevity, the cardiovascular, metabolic and musculoskeletal systems, as well as on mental health and well-being. We outline our scoping review protocol to examine the relationships and effects of golf on physical and mental health.

Methods and analysis Best practice methodological frameworks suggested by Arksey and O'Malley, Levac et $a$ and the Joanna Briggs Institute will serve as our guide, providing clarity and rigour. A scoping review provides a framework to (1) map the key concepts and evidence, (2) summarise and disseminate existing research findings to practitioners and policymakers and (3) identify gaps in the existing research. A three-step search strategy will identify reviews as well as original research, published and grey literature. An initial search will identify suitable search terms, followed by a search using keyword and index terms. Two reviewers will independently screen identified studies for final inclusion. Dissemination We will map key concepts and evidence, and disseminate existing research findings to practitioners and policymakers through peer-reviewed and non-peer reviewed publications, conferences and inperson communications. We will identify priorities for further study. This method may prove useful to examine the relationships and effects of other sports on health.

\section{INTRODUCTION/BACKGROUND}

Health has been defined by the WHO as "a state of complete physical, mental and social well-being and not merely the absence of disease or infirmity." ${ }^{1}$ It is determined by a range of individual behaviours and characteristic factors, and the physical, social and economic environment that people are subject to. ${ }^{2}$

Golf is played by around 55 million people, ${ }^{3}$ representing approximately $1 / 127$ of the global population young and old, in 206 countries worldwide. ${ }^{4}$ Participation in golf affects a number of factors which determine health. Primary research papers $^{5-7}$ and reviews ${ }^{8-10}$ of the literature have described the relationships and effects of golf on aspects of physical health and mental health. These studies have described golf as a valuable form of physical activity, ${ }^{78}$ with beneficial lipid profile cardiovascular and longevity associations ${ }^{5-7}$ and mental health benefits. ${ }^{8}$ Studies have also highlighted musculoskeletal injuries including back, wrist and elbow injuries associated with golf, ${ }^{10} 11$ and accidents, for example, eye and head injuries related to ball and club strikes that can occur in a golfing context. ${ }^{8}{ }^{12}$ However, no study has provided a 'big picture' view evaluating the effects and relationships of golf on physical and mental health. The rationale for this scoping review is to conduct a methodologically rigorous study providing this overview, mapping and summarising the evidence, and identifying significant gaps in the literature.

A key theme to emerge is that golf is recognised as a form of physical activity. Regular physical activity is known to prolong life expectancy and positively impact on many physical and mental health conditions. ${ }^{13}$ The most recent Global Burden of Disease study found physical inactivity to be 1 of the 10 leading causes of death worldwide, while its impact is greater in North America, Europe, Australasia and other areas where golf is commonly played. ${ }^{14}$ The WHO currently labels physical inactivity as the fourth leading cause of death worldwide, ${ }^{15}$ and it has been labelled 'the biggest public health challenge of the 21st Century'. ${ }^{16}$ Physical inactivity is a truly global problem, estimated to be responsible for $>5.3$ million deaths each year, a number greater than obesity or alcohol excess. ${ }^{13}$ Given the potential physical and mental health benefits, and savings estimated at $£ 0.9$ billion ${ }^{17}$ per year to the UK National Health Service alone by increasing physical activity, practitioners and policymakers are increasingly interested in further researching the health effects of particular forms of physical activity. Furthermore, initial studies suggest that knowledge of the health benefits of physical activity positively influences people to be active. ${ }^{18}$ Initial observation of the literature also highlights the potential negative effects of participation in sport and golf in particular, for example, musculoskeletal injuries and accidents. ${ }^{10-12}$ There may also be relationships between golf spectating and health. Golf is unusual as a spectator sport, typically taking place in a few square miles of arena, with $150+$ players to watch, and the opportunity not to be restricted to a designated seat but to walk the course to see the entire arena ${ }^{19}$ or follow players with a potential positive impact on physical activity levels. Hazards for spectators may include being struck by errant golf balls.

A systematic review looking at health benefits related to sport suggested that evidence was strongest for running and football, while ongoing evaluation and research looking at other sports was required. ${ }^{20}$ A systematic review reported the health benefits of cycling for population health. ${ }^{21}$

Preliminary searches of SportDiscus and Google helped to broaden knowledge of existing literature and shape the formulation of our question. Five relevant reviews were identified, none of which were scoping reviews. ${ }^{8-10} 2223$ Most notable was a 
review that looked comprehensively at a range of health outcomes, but did not articulate the methods used. ${ }^{8}$ The remaining articles covered a narrow area in the field of golf and health.

From this process, the primary research question was formulated as 'What is known about the relationships and effects of golf on physical and mental health?' We describe a scoping review protocol that is different to previous studies in broadly assessing this topic, and providing clear and robust methodology to examine the relationship and effects of golf on physical and mental health. Once conducted, the scoping review will map the key concepts and evidence, disseminate existing research findings to practitioners and policymakers through peerreviewed and non-peer reviewed publications, and identify research gaps and priorities for further study. A preliminary search for scoping reviews on this topic has been conducted with no similar study found.

\section{METHODS}

Scoping reviews typically have a broader research question than systematic reviews. Systematic reviews search for the best available evidence to answer a narrower question, while scoping reviews can allow investigators to map the extent of research in a given area and share existing findings. Both systematic and scoping reviews can provide a comprehensive and rigorous approach in assessing the available literature, although scoping reviews focus less on the quality of the research. Owing to the wide scope of the research question, scoping reviews map and articulate the available evidence without a formal analysis of the methods or quality of the studies. They map and draw from all useful evidence, as opposed only to the best available evidence used in systematic reviews.

Of the various approaches available for reviewing published and grey literature, a scoping review was felt to be most appropriate to:

A. Map the key concepts and evidence available;

B. Summarise and share existing research findings with policymakers, practitioners and other relevant stakeholders;

C. Identify research gaps in the existing literature.

The methodological framework presented by Arksey and $\mathrm{O}^{\prime}$ Malley ${ }^{24}$ is well established and frequently used for scoping reviews enabling a clear structure while adding rigour, clarity and reproducibility. Levac et $a l^{25}$ and the Joanna Briggs Institute $^{26}$ have adapted this process, and these adaptations informed our scoping review protocol.

These frameworks also discuss the need for scoping reviews to be iterative, with those which make adaptions to the research questions, based on initial searches producing best results.

We adopted the five-stage process proposed by Arksey and O'Malley. ${ }^{24}$

\section{Stage 1: Identify the research question}

The research question was framed by assimilating themes from the preliminary searches, and opinions were sought from experts in the field of sports medicine and golf. Using a concept, target population and outcomes of interest approach, we formulated a broad research question:

What is known about the relationships and effects of golf on physical and mental health?

\section{Stage 2: Identifying relevant studies Eligibility criteria}

Together, the authors decided on the following inclusion and exclusion criteria to guide the search and review articles found.
Inclusion criteria:

- Research articles are not limited by geographical location, language or setting.

- All age groups and both sexes of participants.

- Research that looks at the general population, as well as at specific population groups (with a specific physical or mental health illness or condition).

- All forms of golf (including but not limited to 18 holes, 9 holes, driving range, spectating).

- Any physical and/or mental health condition.

- Sources of information can include primary research studies, reviews (including but not limited to systematic reviews, scoping reviews, meta-analyses), guidelines, as well as grey literature to include unpublished and ongoing trials, annual reports, dissertations and conference proceedings.

Explicit exclusion criteria identified are:

- Opinion pieces/opinions, magazine and newspaper articles, case reports, papers with no data.

- Health and safety/occupational issues not related to playing or watching golf.

\section{Search strategy and databases}

Step 1: An initial limited search

An initial limited search of SPORTDiscus and Google Advanced Search for review articles was conducted. The search terms used were 'golf' AND 'health' AND 'review'. All 56 studies identified by SPORTDiscus were reviewed, along with the first 200 from the Google search. 26 studies in total proved relevant, with the references from these studies being reviewed for further relevant papers. A search of ProQuest dissertations did not find any similar dissertations.

\section{Step 2: Identify key words and index terms}

The title, the abstract and the index terms used to describe the articles identified in step 1 were analysed. The research team identified golf as the only primary research term. For the healthfocused databases, namely MEDLINE and PsycInfo, "golf" will be used as the only search term to maximise inclusivity. Secondary search terms will include a broader set of keywords for SPORTDiscus, Web of Science and Google Scholar. Boolean terms AND and OR will help to extract relevant studies, while filtering methods will help in applying exclusion criteria. All relevant articles from SPORTDiscus and Web of Science will be reviewed, with the same search strategy applying to Google Scholar with a pragmatic decision to review only the articles with these terms in the title taken following consultation with a research librarian.

A similar strategy will be applied to the grey literature. The same search terms used for SPORTDiscus, Web of Science and Google Scholar will be applied to search for theses in the ProQuest database. "Golf" as the only search term will be used for the WHO International Clinical Trials Registry Platform. The advanced search function on Google will be utilised to look for relevant reports and articles from the World Golf Foundation, the Royal and Ancient, the British Journal of Sports Medicine, The American College of Sports Medicine and the Faculty of Sports and Exercise Medicine while representatives of these organisations will be contacted for further information.

\section{Step 3: Further searching of references and citations}

A search will be conducted of the reference list of relevant identified articles while authors of all relevant primary comprehensive, scoping or systematic reviews will be contacted for further information. 
Scoping reviews are typically iterative, as reviewers become increasingly familiar with the research and evidence, ${ }^{24}$ and thus potentially useful further search terms and sources of information may be incorporated following input from a research librarian.

Search strategies will be documented, and the complete final search strategies are available from the corresponding author. References will be extracted and imported to the Endnote 7 reference management system, where database specific folders will be utilised and duplicates then eliminated.

\section{Stage 3: Study selection}

Titles and abstracts identified by the search strategy will be evaluated against the eligibility criteria by one reviewer (AM). A second reviewer (LD) will complete the same process on a random sample of $10 \%$ of titles and abstracts as a quality check. The reviewers will assess the title and abstracts independently, using the inclusion and exclusion criteria. If the reviewers disagree on the eligibility of a study, it will be discussed with a third reviewer (DA). If a consensus is not reached, the study will be included in the scoping review. If the same inclusion/exclusion decision is taken by both reviewers for over $95 \%$ of studies assessed, then AM will review the titles and abstracts of all other papers. If concordance is less than $95 \%$, then all titles and abstracts will be assessed by both reviewers.

Following this, the full text will be sourced for all articles meeting the inclusion criteria. A PRISMA flow diagram will report numbers once the review has been undertaken.

\section{Stage 4: Charting the data}

Extraction of the results

Charting tables similar to that favoured by the Joanna Briggs Institute $^{26}$ will be used to record and assimilate extracted data from included studies as set out below. The search strategy employed thus far will allow the development of initial a priori categories. Emergent themes will also be charted. Two reviewers (AM and LD) will undertake data extraction duties. A data extraction form will be used to extract details pertaining to study design, methods, participants, interventions and findings. The data extraction strategy will involve AM extracting data from $90 \%$ of included studies and LD extracting data from $10 \%$ of studies. LD will check $10 \%$ of AM's data extractions for accuracy and vice versa. Any disagreements over extracted data will be discussed at group meetings. If significant differences exist, all papers will have data extracted by two reviewers.

A. Author (s)

B. Year of publication

C. Origin (where the study was published/conducted)

D. Aims/purpose

E. Study population and sample size (if applicable)

F. Methodology/methods

G. Intervention type, comparator, details of these (eg, duration of the intervention) (if applicable)

H. Duration of the intervention (if applicable)

I. Outcomes and details of these (eg, how measured, etc) (if applicable)

J. Key findings that relate to the scoping review research questions

Charting results is commonly an iterative process during scoping reviews; if unforeseen useful data can be charted, then further categories of tables may be added or table headings updated if needed.

Where full papers cannot be obtained, efforts to obtain the full paper via hard or electronic copy via the university library will be made. When the paper cannot be found, we will write to the corresponding author to request it. If the full paper cannot be found, the study will not be included.

\section{Stage 5: Collating, summarising and reporting the results}

We envisage that the methods employed in this scoping review protocol will enable us to collate and summarise existing knowledge on this broad topic. On the data being extracted, we will:

A. Map the key concepts and evidence available;

B. Summarise existing research findings;

C. Identify research gaps in the existing literature.

The results will be presented in two parts.

1. A numerical analysis will map the data in tabular and diagrammatic form, showing distribution of studies by theme, period of publication, country of origin and study method.

2. A thematic summary will provide a descriptive analysis describing how the research identified relates to the research question and the main findings from these, organised by theme.

\section{DISSEMINATING AND COMMUNICATING RESULTS}

Ultimately, the scoping review will inform the priority areas for further research, and provide insights into physical and mental benefits and disbenefits associated with golf. Disseminating and communicating these findings widely may help the public and policymakers understand any benefits associated with participation in golf and encourage participation if benefits are found, and also inform on the reduction of risk where disbenefits are highlighted, as well as articulating priority areas for further research.

Findings will be summarised in an account for peer-reviewed publication. A multiplatform approach will be used to help communicate findings with the public and policymakers, the popular and industry press, a newly compiled website (http:// www.golfandhealth.org) and social media including twitter @golfandhealth and facebook 'Golf and Health' utilised as part of a communication plan. Elite female and male professional and former professional golfers, as well as celebrities with an interest in golf, have volunteered to help communicate key findings using the popular press and social media to build awareness of any potential relationships and effects of golf on physical and/or mental health. The results will be reported through these mechanisms regardless of whether negative or positive relationships or effects are reported.

\section{CONCLUSION}

Scoping reviews can be particularly effective for addressing widely framed research questions. This scoping review protocol provides rigour and a framework to enable us to look at the relationships and effects of golf on physical and mental health. It will enable us to map the key concepts and insights available, summarise and share existing research findings with all relevant stakeholders and identify research gaps in the existing literature.

\section{What is known and what this adds}

Scoping reviews can provide a useful framework to collate and summarise information on a broad topic.

- An evidence-informed overview of the effects and relationships of golf on health is currently lacking.

- Golf as a physical activity may have physical and mental health benefits. Potential disbenefits include the risk of musculoskeletal and accidental injuries and skin cancer. 
Scoping reviews provide a useful mechanism for addressing broad questions like the relationships and effects of particular sports on health, and this scoping review protocol may provide a structure that could be utilised by organisations, policymakers and practitioners in other sports or physical activities.

Twitter Follow Andrew Murray at @docandrewmurray and Nanette Mutrie at @nanettemutrie

Acknowledgements The authors wish to thank Karim Khan, editor-in-chief of the British Journal of Sports Medicine, Marshall Dozier, the head librarian for population health at the University of Edinburgh, representatives of the World Golf Foundation, the Royal and Ancient, and the European Tour for their support in identifying suitable studies, and Professor Scott Murray, Paul Kelly and Ruth McQuillan for their advice regarding methodological considerations.

Contributors AM, LG and NM identified the method, while AM and DA identified existing scoping review frameworks to develop this scoping protocol. AM and LD worked with the team librarian (MD) to develop search terms and a search strategy. All authors have contributed to the development of the research questions and study design. All authors developed the first and subsequent drafts of the manuscript and also reviewed and approved the manuscript.

Funding This work was supported by an unrestricted grant from the World Golf Foundation; Medical Research Council.

Competing interests AM reports an unrestricted grant from the World Golf Foundation. RH and AM receive personal fees from the European Tour Golf for clinical work.

Provenance and peer review Not commissioned; externally peer reviewed.

Open Access This is an Open Access article distributed in accordance with the Creative Commons Attribution Non Commercial (CC BY-NC 4.0) license, which permits others to distribute, remix, adapt, build upon this work non-commercially, and license their derivative works on different terms, provided the original work is properly cited and the use is non-commercial. See: http://creativecommons.org/ licenses/by-nc/4.0/

\section{REFERENCES}

1 World Health Organisation. Preamble to the constitution of the World Health Organisation as adopted by the International Health Conference. World Health Organisation, 1946.

2 World Health Organisation. Health impact assessment- the determinants of health World Health Organisation, 2011

3 Farrally MR, Cochran AJ, Crews DJ, et al. Golf science research at the beginning of the twenty-first century. I Sports Sci 2003;21:753-65.

4 The Royal and Ancient. Golf Around the World. Online. 2015;1.

5 Parkkari J, Natri A, Kannus P, et al. A controlled trial of the health benefits of regular walking on a golf course. Am J Med 2000;109:102-8.
6 Farahmand B, Broman G, de Faire U, et al. Golf: a game of life and death-reduced mortality in Swedish golf players. Scand J Med Sci Sports 2009;19:419-24.

7 Broman G, Johnsson L, Kaiiser L. Golf: a high intensity interval activity for elderly men. Aging Clin Exp Res 2004;16:375-81.

8 Walker Research Group. World golf foundation and golf 20/20 commission report on golf's health benefits. Walker Research Group, 2011.

9 McHardy A, Pollard H, Luo K. Golf injuries: a review of the literature. Sports Med 2006:36:171-87.

10 Cabri J, Sousa JP, Kots M, et al. Golf-related injuries: a systematic review. Eur J Sport Sci 2009;9:353-66.

11 Batt ME. A survey of golf injuries in amateur golfers. Br I Sports Med 1992;26:63-5.

12 Takatama A. Eleven cases of eye injury while playing golf. Jpn I Clin Opthalmol 2002;56:567-70.

13 Lee IM, Shiroma EJ, Lobelo F, et al. Effect of physical inactivity on major non-communicable diseases worldwide: an analysis of burden of disease and life expectancy. Lancet 2012;380:219-29.

14 Lim SS, Vos T, Flaxman AD, et al. A comparative risk assessment of burden of disease and injury attributable to 67 risk factors and risk factor clusters in 21 regions, 1990-2010: a systematic analysis for the Global Burden of Disease Study 2010. Lancet 2012;380:2224-60.

15 Organisation WH. Global recommendations on physical activity for health. World Health Organisation, 2010.

16 Blair SN. Physical inactivity: the biggest public health problem of the 21st century. Br J Sports Med 2009;43:1-2.

17 Scarborough P, Bhatnagar P, Wickramasinghe KK, et al. The economic burden of ill health due to diet, physical inactivity, smoking, alcohol and obesity in the UK: an update to 2006-07 NHS costs. J Public Health (Oxf) 2011;33:527

18 Affuso O, Cox TL, Durant NH, et al. Attitudes and beliefs associated with leisure-time physical activity among African American adults. Ethn Dis 2011;21:63-7.

19 Hansen H, Gauthier R. Spectators' views of LPGA golf events. Sport Mark Q 1993;2:17-25.

20 Oja P, Titze S, Kokko S, et al. Health benefits of different sport disciplines for adults: systematic review of observational and intervention studies with meta-analysis. Br I Sports Med 2015;49:434-U34.

21 Oja P, Titze S, Bauman A, et al. Health benefits of cycling: a systematic review. Scand J Med Sci Sports 2011;21:496-509.

22 Thériault G, Lachance P. Golf injuries. An overview. Sports Med 1998;26:43.

23 Brunette DD. A good walk spoiled. Golf's links to medicine. Minn Med 1997;80:25-30.

24 Arksey H, O'Malley L. Scoping studies: towards a methodological framework. Int I Soc Res Methodol 2005;8:19-32.

25 Levac D, Colquhoun H, O'Brien KK. Scoping studies: advancing the methodology. Implement Sci 2010;5:1-9.

26 Peters M, Godfrey C, Mclnerney P, et al. Guidance for conducting systematic scoping reviews. Int J Evid Based Healthc 2015;13:141-6. 